\title{
Configuration of daylighting system via fibers and experiments of concentrated sunlight transmission
}

\author{
Song $\mathrm{JF}^{1, *}$, Yang $\mathrm{YP}^{1}$, Hou $\mathrm{HJ}^{1}$, Zhang $\mathrm{MX}^{1}$ \\ ${ }^{1}$ The New and Renewable Energy of Beijing Key Laboratory, North China Electric Power University, Beijing, \\ 102206, China \\ * Corresponding author. Tel: +86-10-61772816, Fax: +86-10-61772816, E-mail: songjifeng@ncepu.edu.cn
}

\begin{abstract}
A daylight concentrating and transmission system via plastic fibers based on dual axis sun tracker to lighting indoor has been built and investigated. The sunlight tracking and concentrating platform adopting horizontal coordinate system combined with photosensitive sensor can realize high position resolution. A sunlight concentrating and transmission experiment has been carried out using 6m long PMMA plastic fibers based on that platform. It is found that color temperature of light transmitted by fibers is $600 \mathrm{~K}$ lower than that of nature light. The spectrum of light transmitted by fibers is similar to that of nature light. This similarity also exists in chromaticity coordinate, color rendering index, dominant wavelength between light transmitted by fibers and nature light. A quantitative determination of flux loss has been carried out and the results show that there is an attenuation about $2 \mathrm{db}$ existing on the interface of fiber.
\end{abstract}

Keywords: Sun tracking, Fibers, Concentrated, Transmission

\section{Nomenclature (Optional)}

NA numerical aperture

$\alpha$ altitude angle

$\gamma$ azimuth angle.

$\varphi$ latitude

$\delta$ declination

$P_{\text {out }}$ output light flux.................................... Iumen

$P_{\text {in }}$ input light flux................................. lumen

$\tau$ time adjustment.......................................s

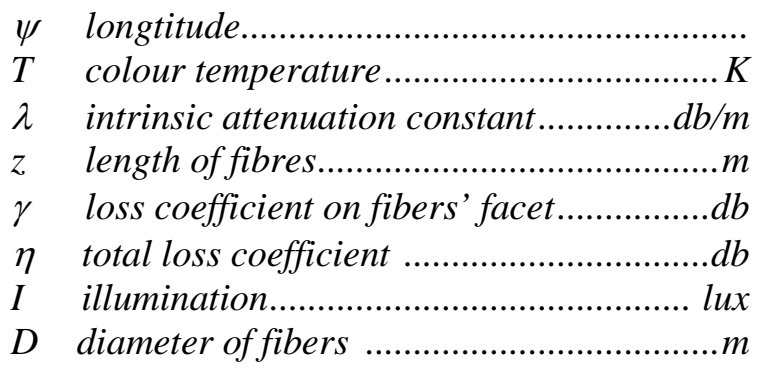

\section{Introduction}

The daylighting system is an optic-mechanical-electric technology that collects day light outside to transmit into basement and room lacking nature light by fibers in high concentrated level [1-2]. The infrared portion of solar radiation has been separated and eliminated by lens and fibers so that the output flux is a cool light. There are two major benefits from daylighting. The first benefit is the reduction in purchased electricity needed to light the building, and the second benefit is a reduced cooling load due to the high efficiency of light. Another potential benefit of daylighting is the advantageous factors for healthy of natural lighting but this effect is difficult to quantify. The excellent color rendering properties of daylight and its close match to the photopic response of the human eye make it an ergonomic light source that is generally preferred for pleasant working conditions[3-4]. There are two types of daylighting systems including light guide pipe and fibers. The latter is the research hotspot at present due to its smaller and few penetrations on the roof which means saving on the building's heating, cooling, and maintenance bills. The purpose of this particular study is to evaluate the feasibility and performance of the technology. The day light concentrating and transmission system via fibers consists of a two-axis sun tracker and concentrating collector that gather direct normal solar radiation into the fibers. The key to realize stable flux output is high precision sun tracking which need a trade-off with fabrication cost[6]. And, at the present time, the optical parameters of sunlight transmission system via fibers have not get adequacy quantitative determination. Those problems provide the investigation motivation of this 
research work. According to the structure forms, there are two ways of focalization including optical fiber bundle and single fiber. For the former, concentrator with large diameter is used to produce large focal spot, and so it can use optical fiber bundle to receive the concentrated radiation[7-9]. For the latter, the concentrator is lens with small diameter which produces small focal spot only suiting for single fiber. It is critical to obtain high accuracy position tracking in respect that small focus error will cause a large amplitude reduction of out flux due to single fiber's small diameter. Although much research has been devoted into sunlight concentrating and transmission system via fibers, there are still lots of problems and unknown characterizes awaiting solutions, such as quantitative study of the spectrum of output light. In order to try to answer above questions, a dual-axis sun tracking system has been investigated by the combined use of horizontal coordinate system and photosensitive sensor designed specially, based on that corresponding research has been done.

\section{Dual axis sun tracking and concentrating system}

\subsection{Configuration of hardware}

The system mainly is composed of support, reducing gears, motor, lens and control module. It must be emphasized that small deviation of focusing spot will lead to significant instability attributed to no light preserved module existing in the daylighting system. To satisfy above strict demand, orbit calculation method and optical sensing method are integrated into control flow. The prototype of the dual axis tracking and concentrating system is shown in Fig 1.

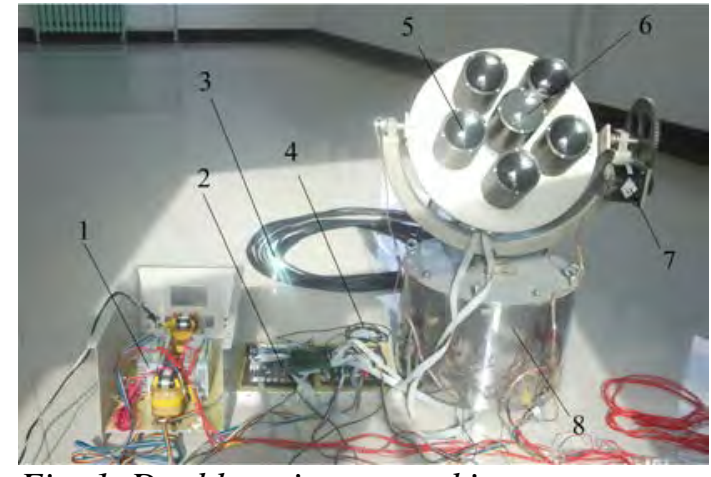

Fig. 1. Double-axis sun tracking system

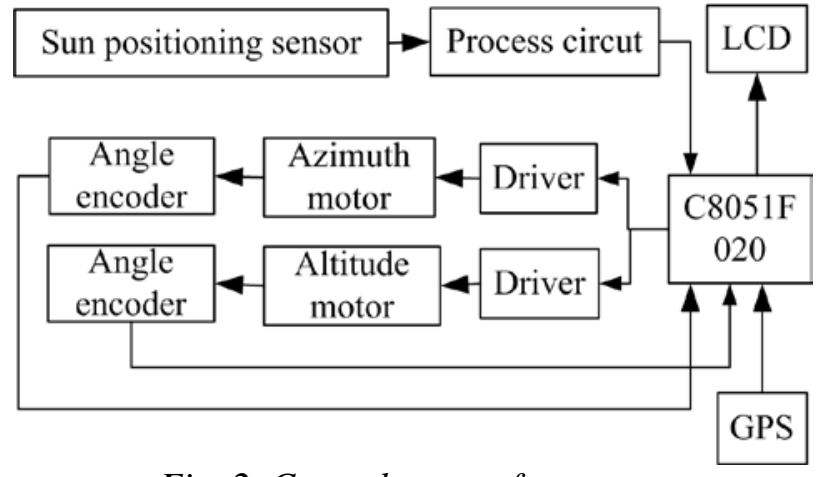

Fig. 2. Control system frame

In fig.1, the components numbered are step motor drivers, control board, plastic fibers, GPS module, lens, sun positioning sensor, altitude motor, substructure for installing azimuth motor successively. In practical, it is too difficult to realize accuracy positioning about $0.1^{\circ}$ only depending on orbit calculation due to the varied limits, such as installation error and gravity deformation, et al, although it is possible in theory. The misalignment between geodetic coordinate and device coordinate led by installation error and gravity deformation will result in an inevitable calculation error in sun position[10]. So an optical sun positioning sensor is designed specially to eliminate the error accumulation and initial error, which uses photosensitive elements array to sensing the location of the focusing spot generated by lens. By comprehensive utilization of two methods, it is able to realize stability and high precision profit from orbit calculation method and sun optical positioning sensor respectively. The control system is designed to work automatically. Step motors are used to drive the tracking action and angle encoders are applied to feedback the real angle information of the mechanical components. Global positioning system module is adopted to provide exact time and latitude and longitude which are the parameters to calculate the sun position. With the help of GPS module, the tracking system can figure out the sunrise and sunset time to realize full automatic tracking without manual operation, meaning remarkable reduction of 
maintenance. For convenience, a LCD display screen is installed to show real-time working state. Plano convex lenses made of K9 optical glass are adopted to concentrate sunlight which has a transmission coefficient that is no less than 0.9 at visible spectrum range. It is no cooling problem for lenses and fibers because there is nonexistence of hot spots due to their high transparency. Performance parameters of the hardware have been described in tab 1 . To facilitate the precision drive, sinusoid subdivision drivers are used to improve step motors to achieve 12800 pul ses per cycle, about $0.028^{\circ} /$ pulse. In addition, gear pairs are applied to further improve the fine adjustment; however nonlinearity caused by gear clearance emergences isochronously. To ensure stable tracking, it is needed to introduce intelligent algorithm to compensate the nonlinearity. For instance, database is recorded in the program memory to distinguish different situations of nonlinearity. The concentrator is convex lens made of super white glass which transmittance is 0.92 . According to the sun's

Table.1 Parameters of double-axis sun tracking and concentrating platform

\begin{tabular}{llll}
\hline Name & Unit & Amount & illustration \\
\hline Torque of azimuth motor & N.m & 3.6 & Step motor \\
Torque of altitude motor & N.m & 1.2 & Step motor \\
Reduction ratio of azimuth & & $1: 3$ & Straight gear \\
Reduction ratio of altitude & & $1: 5$ & Straight gear \\
Tracking type & & & Orbit and optical sensor \\
Tracking accuracy & $\circ$ & \pm 0.15 & \\
Range of altitude & $\circ$ & $0 \sim 90$ & \\
Range of azimuth & $\circ$ & $0 \sim 360$ & \\
Diameter of lens & $\mathrm{m}$ & $\Phi 0.1$ & K9 material \\
Focal length & $\mathrm{mm}$ & 180 & Adjustable \\
Concentration ration & & $900-10000$ & Adjustable \\
Transmittance of lens & & 0.92 & Visible band \\
\hline
\end{tabular}

\subsection{Control method}

As for the optical sun positioning sensor, it is used to detect detailed deviation and feedback the signal to microcontroller to realize exact tracking. The most important advantage of the optical sun positioning sensor is the ability to eliminate the error accumulation caused by errors from motor or reducing gears. The optical sun positioning sensor is good at dealing with error accumulation but bad at anti-climate impacts while the orbit calculation method is opposite. So it is wise to adopt combined utilization of both methods to obtain good tracking accuracy and anti-interference ability meanwhile. The flow chart of sun tracking process is shown in Fig 3. B ecause of complexity in practice, the flow chart has more fine regulation actions than that listed out in Fig 3. 


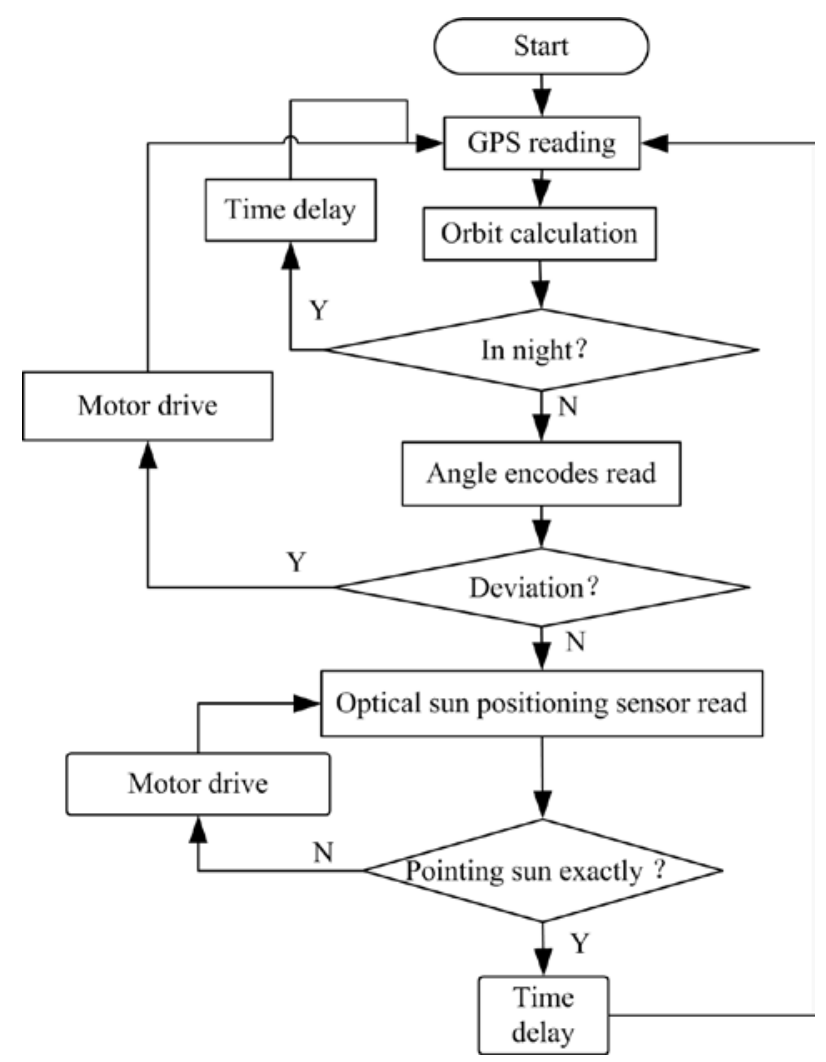

Fig.3. Control flow chart of sun tracking process

\section{Plastic fibers}

The diameter of fibers used for concentrated sunlight transmission is flexible and two orders of magnitude higher than that of fibers used for distant communication, whose range is 1$6 \mathrm{~mm}$ generically. The materials of fibers include polymethyl methacrylate(PMMA), polystyrene and special quartz. Light attenuation is severe, about $0.25 \mathrm{db} / \mathrm{m}$, only suiting for close distance transmission not longer than $30 \mathrm{~m}$. Some parameters of the fibers tested in the work are described in Tab 2. The fiber tested in this research is made of PMMA, and has absorption peaks in $620 \mathrm{~nm}$ and $705 \mathrm{~nm}$ which is shown in Fig 4. The advantages of PMMA fibers are flexible and big numerical aperture which is very in favor for light focusing. Unfortunately, the upper limit for work temperature is only $70^{\circ} \mathrm{C}$ which restricts the upper limit of concentration ratio, about 2500 without water cooling. But this upper limit could be extended to 10000 for quartz fiber which is used in solar furnace.

Table. 2 Parameters of PMMA fibers

\begin{tabular}{lccl}
\hline Name & Unit & Amount & illustration \\
\hline Diameter & $\mathrm{mm}$ & 2.5 & \\
Length & $\mathrm{m}$ & 6 & \\
Numerical aperture & & 0.5 & Ranger of angle of incidence is $\pm 30^{\circ}$ \\
Average attenuation & $\mathrm{db} / \mathrm{m}$ & 0.25 & $380 \mathrm{~nm} \sim 760 \mathrm{~nm}$ \\
\hline
\end{tabular}




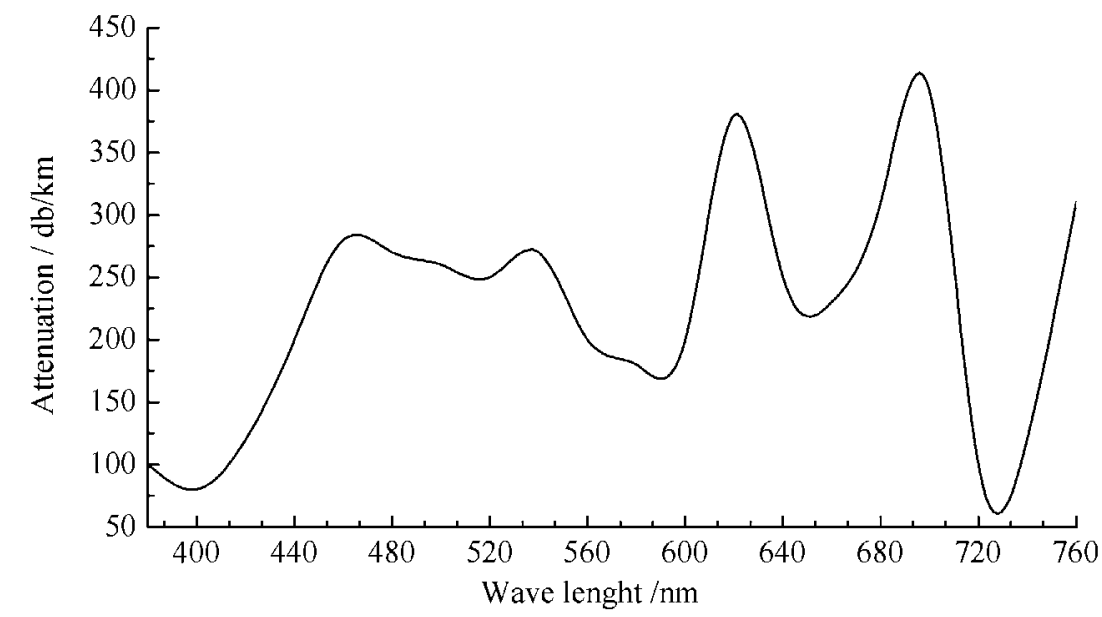

Fig.4. Curve of intrinsic attenuation of PMMA fibers

\section{Results}

The experimental system consists of the dual axis tracking and concentrating system, fibers, illuminometer and HAAS-2000 spectral radiometer, as in Fig 5. A sunlight concentrating and transmission experiment lasted for 9 hours has been carried out using $6 \mathrm{~m}$ long PMMA fibers .

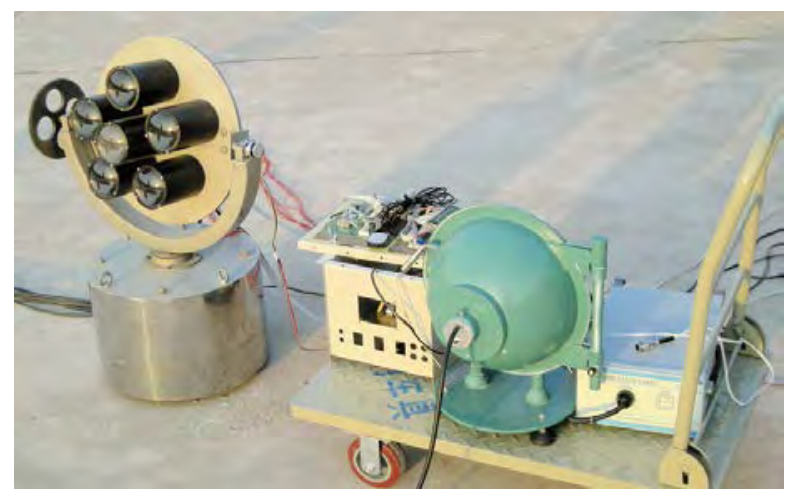

Fig.5 Optic testing system of concentrated sunlight transmission system

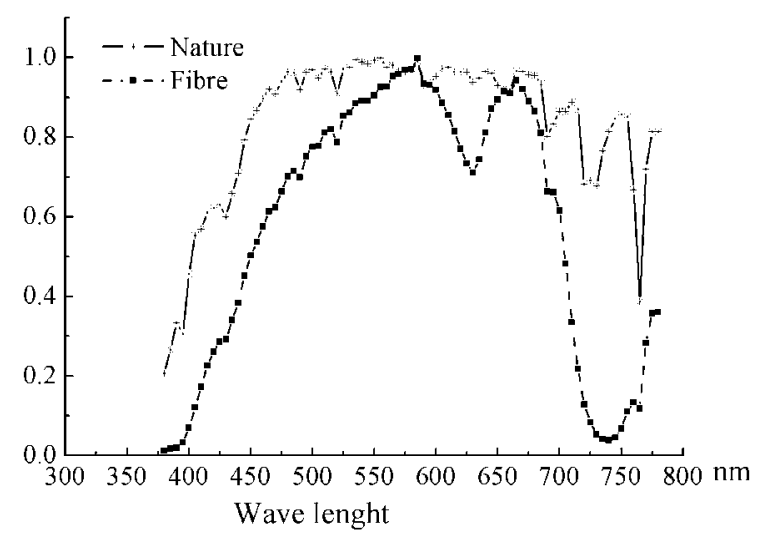

Fig.6 Spectrum comparison of nature light and light transmitted by fibers in visible band

The spectrum of light transmitted by fibers is similar to that of nature light, as shown in Fig 6 . Although the deviation between two curves is evidence in $705 \mathrm{~nm}$ led by absorption peaks of fibers, it influences the visual perception slightly because it is away from green band which is most sensitive for eyes. This similarity also exists in chromaticity coordinate, color rendering 
index, dominant wavelength between light transmitted by fibers and nature light, as described in Tab 3.

Table.3 Parameters comparison between nature light and light transmitted by fibers

\begin{tabular}{llll}
\hline Parameters & Light out fibers & Daylight & Difference \\
\hline Color temperature & $4444 \mathrm{~K}$ & $5037 \mathrm{~K}$ & $-11.8 \%$ \\
Dominant wave length & $571.6 \mathrm{~nm}$ & $569.4 \mathrm{~nm}$ & $+0.4 \%$ \\
Peak wavelength & $585 \mathrm{~nm}$ & $538 \mathrm{~nm}$ & $+8.7 \%$ \\
Width of half wave & $255.8 \mathrm{~nm}$ & $360.6 \mathrm{~nm}$ & $-29 \%$ \\
Color coordinate X & 0.3688 & 0.3446 & $+7 \%$ \\
Color coordinate Y & 0.3972 & 0.357 & $+11.2 \%$ \\
Color rendering index & 88.9 & 99.3 & $-10.5 \%$ \\
Red light ratio & 17.9 & 18.8 & $-4.8 \%$ \\
\hline
\end{tabular}

As identified in Tab 3, the flux transmitted through fibers is approximate to nature light, so it can satisfy the need of drawing office, indoor plant cultivation and shady bedroom.

As for the transmission loss, it is consists of three parts which are intrinsic loss, loss on end faces of fibers and loss led by bend. Among them, the intrinsic loss is a constant while loss on face is a large variation, determined by roughness mainly. In fact, the loss on face takes an essential ratio of total loss. So it makes sense to make certain that how much loss on face is. A experiment for determining the value of loss on face is designed, which separates the intrinsic loss from total loss by a series calculations.

For fibers without bend, we have

$P_{\text {out }}(z)=P_{\text {in }}(0) e^{-\lambda z} e^{-\gamma}$

Here $z=6 m$. As for the total $\eta$, it can be described as followed

$\eta=10 \lg \frac{P_{\text {out }}(z)}{P_{\text {in }}(0)}=-10(\lambda z+\gamma) \lg e$

Traditionally it is a custom to use decibel in attenuation analysis, so we have

$\left\{\begin{array}{l}\bar{\lambda}=(10 \lg e) \cdot \lambda \\ \bar{\gamma}=(10 \lg e) \cdot \gamma\end{array}\right.$

It is easy to get following conclusion after a further derivation

$\left\{\begin{array}{l}\eta=-\bar{\lambda} z-\bar{\gamma} \\ \bar{\gamma}=-\eta-\bar{\lambda} z\end{array}\right.$

$\bar{\lambda}$ is a constant known as $0.25 \mathrm{db} / \mathrm{m}$ and $P_{\text {out }}(\mathrm{z})$ can be measured by integrating sphere and spectroradiometer while $P_{i n}(0)$ can be worked out by the following formula 
$P_{\text {in }}(0)=I \cdot \pi D^{2} / 4$

Measured data of luminance outdoor and output flux of fibers is shown in Fig 7. Two curves are anastomotic through one day.

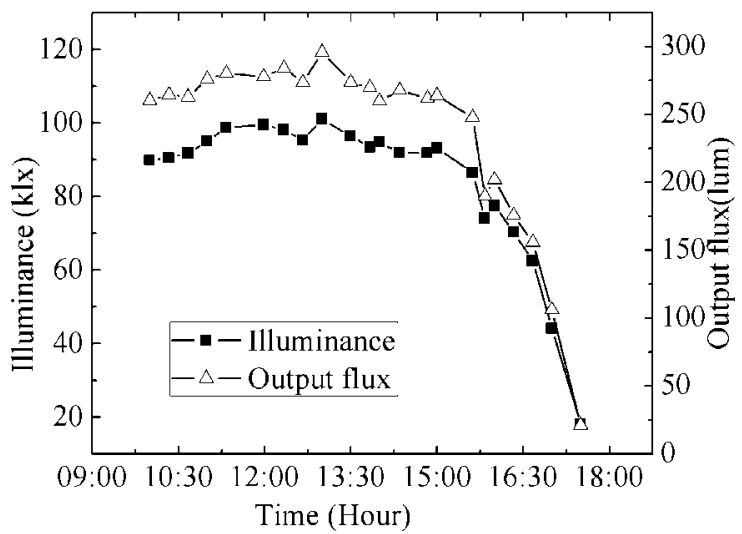

Fig.7 Corresponding relation of illumination outside and output flux of fibers

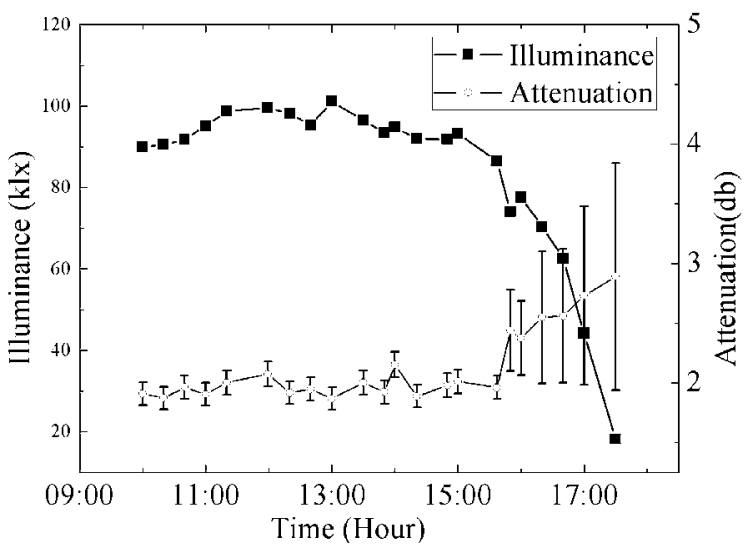

Fig.8 Corresponding relation of illumination outside and attenuation on interface

From measured data recorded in Fig7, the loss on face can be calculated out as shown in Fig 8 . The loss on face can be regarded as a constant about $2 \mathrm{db}$ approximately which is accord with the optical principle. As to the increasing after $16: 00 \mathrm{pm}$, it can be explained that scattering radiation takes more and more proportion in total sunlight and this scattering radiation cannot be focusing onto the fibers, so above formulas in this situation produce large result.

\section{Conclusions}

Combination of orbit calculation method and sun optical positioning sensor posses accuracy and stability simultaneously in sun tracking which is the key to ensure the output flux of fibers stable. Different from solar thermal and photovoltaic application, there are no measures to storage nature light and so it is fatal when tracking error exceeds the allowable range. It must be pointed out that low speed drive is very beneficial to obtain high precision positioning in sun tracking which affords more time for the microcontroller to analyze the tracking status and implement compensation motions. This strategy makes full use of the feature of sun slow-moving. It is also relatively economic to adopt low power motors owe to the features of this strategy. 
The spectrum of light transmitted by fibers is similar to that of nature light which make it favorable for places needing daylighting. Color temperature of the output light transmitted by fibres is about $600 \mathrm{~K}$ lower than that of nature light which is caused by the selective absorption of plastic fibers. Fortunately, this selective absorption does not cause severe influence to the optical quality of sunlight transmission. The color rendering index still keeps a high value about 88.9 which is far better than those of incandescent lamp, fluorescent lamp and white LED. What's more, the loss on face of fibers cannot be neglected which is about $2 \mathrm{db}$ that is meaning $40 \%$ loss. The loss on face is relative to the roughness and incident angle. It requires precise measurement and mathematical modeling to establish the quantitative description of concentrated sunlight transmission.

\section{Acknowledge}

Financial support from National Natural Science Foundation of China (No.61004084) and Fundamental Research Funds for the Central Universities (09QG18) and State Key Development Program of (for) Basic Research of China (No. 2009CB219801) are appreciated.

\section{References}

[1] C. Kandilli, K. Ulgen, Review and modelling the systems of transmission concentrated solar energy via optical fibres[J], Renewable and Sustainable Energy Reviews, Volume 13, Issue 1, January 2009, Pages 67-84.

[2] Cemil Sungur, Multi-axes sun-tracking system with PLC control for photovoltaic panels in Turkey[J], Renewable Energy, Volume 34, Issue 4, April 2009, Pages 1119-1125.

[3] Schlegel, G.O., et al., Analysis of a full spectrum hybrid lighting system[J]. Solar Energy, 2004. 76(4): p.359-368.

[4] Tzempelikos, A., A.K. Athienitis, and P. Karava, Simulation of facade and envelope design options for a new institutional building[J]. Solar Energy, 2007. 81(9): p. 10881103.

[5] Teolan Tomson. Discrete two-positional tracking of solar collec-tors Original Research Article, Renewable Energy, Volume 33, Issue 3, March 2008, Pages 400-405.

[6] Tekelioglu, M. and B.D. Wood, Solar light transmission of po-lymer optical fibers. Solar Energy, 2009. 83(11): p. 2039-2049.

[7] Tsangrassoulis, L. Doulos, M. Santamouris, et al, On the energy efficiency of a prototype hybrid daylighting system[J], Solar Energy, Volume 79, Issue 1, July 2005, Pages 56-64.

[8] G. O. Schlegel, F. W. Burkholder, S. A. Klein, et al., Analysis of a full spectrum hybrid lighting system[J], Solar Energy, Volume 76, Issue 4, April 2004, Pages 359-368.

[9] Earl, D.D., Maxey, C.L., Muhs, J.D., 2003. Performance of new hybrid solar lighting luminaire design[J]. In: International Solar Energy Conference, Kohala Coast, Hawaii Island, March 15-18.

[10] H.Arbab, B.Jazi,M.Rezagholizadeh, A computer tracking system of solar dish with twoaxis degree freedoms based on picture processing of bar shadow $[\mathrm{J}]$, Renewable Energy, Vol34(2009) 1114-1118. 